\title{
Chiral magnetic effect and holography
}

\author{
Ingo Kirsch* \\ DESY Hamburg, Theory Group, Notkestrasse 85, D-22607 Hamburg, Germany \\ E-mail: ingo.kirschedesy.de
}

\section{Tigran Kalaydzhyan}

DESY Hamburg, Theory Group, Notkestrasse 85, D-22607 Hamburg, Germany

E-mail: Eigran.kalaydzhyanddesy.de

\begin{abstract}
The chiral magnetic effect (CME) is a highly discussed effect in heavy-ion collisions stating that, in the presence of a magnetic field $B$, an electric current is generated in the background of topologically nontrivial gluon fields. We present a holographic (AdS/CFT) description of the CME in terms of a fluid-gravity model which is dual to a strongly-coupled plasma with multiple anomalous U(1) currents. In the case of two U(1) charges, one axial and one vector, the CME formally appears as a first-order transport coefficient in the vector current. We will holographically compute this coefficient at strong coupling and compare it with the hydrodynamic result. Finally, we will discuss an anisotropic variant of the model and study a possible dependence of the CME on the elliptic flow coefficient $v_{2}$.
\end{abstract}

Xth Quark Confinement and the Hadron Spectrum,

October 8-12, 2012

TUM Campus Garching, Munich, Germany

\footnotetext{
* Speaker.
} 


\section{Introduction}

The chiral magnetic effect (CME) is a hypothetical phenomenon which states that, in the presence of a magnetic field $B$, a nonzero axial charge density will lead to an electric current along the direction of the $B$ field $[\mathbf{U}]$. While there is common agreement that the chiral magnetic effect may occur in off-central heavy-ion collisions, it is still under intense discussion whether or not current measurements of charge-dependent correlation functions are sensitive enough to the CME, see [వ] for a phenomenological analysis. Regardless of how the CME will eventually manifest itself in heavy-ion collisions, we discuss both a hydrodynamical as well as a holographic (AdS/CFT) model for the CME. The fluid-gravity duality seems to be the right framework for a holographic description, as it captures the response of the system to an external perturbation, in case of the CME the perturbation by a $B$ field. In this talk we review the fluid-gravity model proposed in [B], 团] and derive the CME (and related effects) from it. Other holographic approaches to the CME can be found in [1] $-[$ [ए]].

\section{Chiral Magnetic Effect in Hydrodynamics}

\subsection{Hydrodynamics with $n$ anomalous $U(1)$ charges}

The hydrodynamic regime of isotropic relativistic fluids with triangle anomalies has been studied in [[1]]-[]]. Such fluids typically contain $n$ anomalous $U(1)$ charges which commute with each other. The stress-energy tensor $T^{\mu v}$ and $n U(1)$ currents $j^{a \mu}(a=1, \ldots, n)$ are

$$
T^{\mu v}=(\varepsilon+P) u^{\mu} u^{v}+P g^{\mu v}+\tau^{\mu v}, \quad j^{a \mu}=\rho^{a} u^{\mu}+v^{a \mu},
$$

where $\rho^{a}, \varepsilon$, and $P$ denote the charge densities, energy density and pressure, respectively. $\tau^{\mu v}$ and $v^{a \mu}$ denote higher-gradient corrections, and $g_{\mu \nu}$ is the metric with signature $(-,+,+,+)$.

The corresponding hydrodynamic equations are

$$
\partial_{\mu} T^{\mu v}=F^{a v \lambda} j_{\lambda}^{a}, \quad \partial_{\mu} j^{a \mu}=C^{a b c} E^{b} \cdot B^{c},
$$

where $E^{a \mu}=F^{a \mu v} u_{v}, B^{a \mu}=\frac{1}{2} \varepsilon^{\mu v \alpha \beta} u_{v} F_{\alpha \beta}^{a}$ are electric and magnetic fields, and $F_{\mu \nu}^{a}=\partial_{\mu} A_{v}^{a}-$ $\partial_{v} A_{\mu}^{a}$ are the gauge field strengths. The anomaly coefficients are given by a totally symmetric rank-3 tensor $C^{a b c}$. As in [ㅍ] , we expand the constitutive equations for $T^{\mu v}$ and $j^{\mu}$ up to first order, taking $A_{\mu}^{a} \sim O\left(p^{0}\right)$ and $F_{\mu \nu}^{a} \sim O(p)$. The gauge fields $A_{\mu}^{a}$ are non-dynamical.

The response of the system to the application of external magnetic fields and rotation is measured by the chiral magnetic and vortical conductivities $\xi_{B}^{a b}$ and $\xi_{\omega}^{a}$, respectively. The first-order correction of the $U(1)$ currents is given by

$$
v^{a \mu}=\xi_{\omega}^{a} \omega^{\mu}+\xi_{B}^{a b} B^{b \mu},
$$

where $\omega^{\mu} \equiv \frac{1}{2} \varepsilon^{\mu \nu \lambda \rho} u_{v} \partial_{\lambda} u_{\rho}$ is the vorticity. The conductivities $\xi_{\omega}^{a}$ and $\xi_{B}^{a b}$ were first introduced in [2], [1]] and are given by [ए], [2]]

$$
\begin{aligned}
& \xi_{\omega}^{a}=C^{a b c} \mu^{b} \mu^{c}-\frac{2}{3} \rho^{a} C^{b c d} \frac{\mu^{b} \mu^{c} \mu^{d}}{\varepsilon+P}+\mathscr{O}\left(T^{2}\right), \\
& \xi_{B}^{a b}=C^{a b c} \mu^{c}-\frac{1}{2} \rho^{a} C^{b c d} \frac{\mu^{c} \mu^{d}}{\varepsilon+P}+\mathscr{O}\left(T^{2}\right),
\end{aligned}
$$


where $\mu^{a}$ are the corresponding chemical potentials. The terms in $\mathscr{O}\left(T^{2}\right)$ are related to gravitational anomalies [23], which we do not discuss here.

\subsection{Chiral magnetic and vortical effect $(n=2)$}

Physically, the most interesting case is that involving two charges $(n=2)$ [एव, [20, []]. The chiral magnetic effect [四] can be described by one axial and one vector $U(1)$, denoted by $U(1)_{A} \times U(1)_{V}$. A convenient notation for the gauge fields and currents is $(a, b, \ldots=1,2)$

$$
\begin{aligned}
A_{\mu}^{A} & =A_{\mu}^{1}, & A_{\mu}^{V} & =A_{\mu}^{2}, \\
j_{5}^{\mu} & =j^{1 \mu}, & j^{\mu} & =j^{2 \mu} .
\end{aligned}
$$

Let us now derive the chiral magnetic and vortical effects from (2.4) and ([2.5)). $C$-parity allows for $C^{111} \neq 0$ and $C^{122}=C^{221}=C^{212} \neq 0$, while $C^{121}=C^{211}=C^{112}=C^{222}=0$ [四]. The hydrodynamic equations (2.2) then imply non-conserved vector and axial currents,

$$
\begin{aligned}
& \partial_{\mu} j^{\mu}=-\frac{1}{4}\left(C^{212} F_{\mu v}^{A} \tilde{F}^{V \mu v}+C^{221} F_{\mu \nu}^{V} \tilde{F}^{A \mu v}\right), \\
& \partial_{\mu} j_{5}^{\mu}=-\frac{1}{4}\left(C^{111} F_{\mu v}^{A} \tilde{F}^{A \mu v}+C^{122} F_{\mu v}^{V} \tilde{F}^{V \mu v}\right),
\end{aligned}
$$

where we rewrote $E^{b} \cdot B^{c}=-\frac{1}{4} F_{\mu v}^{b} \tilde{F}^{c \mu v}$ (with $\tilde{F}^{a \mu v}=\frac{1}{2} \varepsilon^{\mu v \rho \sigma} F_{\rho \sigma}^{a}$ ).

To restore the conservation of the vector current, we add the Bardeen currents

$$
j_{B}^{\mu}=c_{B} \varepsilon^{\mu \nu \lambda \rho}\left(A_{v}^{V} F_{\lambda \rho}^{A}-2 A_{v}^{A} F_{\lambda \rho}^{V}\right), \quad j_{5, B}^{\mu}=c_{B} \varepsilon^{\mu \nu \lambda \rho} A_{v}^{V} F_{\lambda \rho}^{V}, \quad\left(c_{B}=-C^{122} / 2\right)
$$

to the vector and axial currents,

$$
j^{\prime \mu} \equiv j^{\mu}+j_{B}^{\mu}, \quad j_{5}^{\prime \mu} \equiv j_{5}^{\mu}+j_{5, B}^{\mu} .
$$

Setting also $C^{111}=C^{122} \equiv C / 3$, the hydrodynamic equations (2.2) become

$$
\begin{aligned}
\partial_{\mu} T^{\mu v} & =F^{V v \lambda} j_{\lambda}^{\prime}+F^{A v \lambda} j_{5 \lambda}^{\prime}, \\
\partial_{\mu} j^{\prime \mu} & =0, \\
\partial_{\mu} j_{5}^{\prime \mu} & =C E \cdot B+(C / 3) E_{5} \cdot B_{5} .
\end{aligned}
$$

Using the derivative expansion

$$
j^{\prime \mu}=\rho u^{\mu}+\kappa_{\omega} \omega^{\mu}+\kappa_{B} B^{\mu}+\kappa_{5, B} B_{5}^{\mu},
$$

where $\kappa_{\omega} \equiv \xi_{\omega}^{2}, \kappa_{B} \equiv \xi_{B}^{22}$ and $\kappa_{5, B} \equiv \xi_{B}^{21}$, we obtain from (R.4) and (R.5]) the conductivities ${ }^{1}$

$$
\kappa_{\omega}=2 C \mu_{5}\left(\mu-\frac{\rho}{\varepsilon+P}\left[\mu^{2}+\frac{\mu_{5}^{2}}{3}\right]\right), \quad \kappa_{B}=C \mu_{5}\left(1-\frac{\mu \rho}{\varepsilon+P}\right) .
$$

where $\mu_{5} \equiv \mu^{1}, \mu \equiv \mu^{2}$. There are analogous transport coefficients in the axial current $j_{5}^{\mu}$ [[]]. The axial fields $E_{5 \mu}$ and $B_{5 \mu}$ are not needed and can now be switched off. The first term in $\kappa_{B}$ and $\kappa_{\omega}$, $\kappa_{B}=C \mu_{5}$ and $\kappa_{\omega}=2 C \mu \mu_{5}$, is the leading term in the chiral magnetic (CME) [W] and chiral vortical effect (CVE) [24], respectively. The second term proportional to $\rho /(\varepsilon+P)$ actually depends on the dynamics of the fluid. ${ }^{2}$

\footnotetext{
${ }^{1} \kappa_{5, B}$ represents another effect, which occurs only in the presence of an axial $B$ field [䧃].

${ }^{2}$ In [ㅈ] this term was considered as a one-loop correction in an effective theory and $(\varepsilon+P) / \rho$ was interpreted as the corresponding infrared cutoff in the energy/momentum integration.
} 


\section{Fluid-gravity model for the chiral magnetic effect}

In this section we construct the gravity dual of an isotropic fluid with $n$ anomalous $U(1)$ charges. We start from a five-dimensional $U(1)^{n}$ Einstein-Maxwell theory in an asymptotic AdS space. The action is

$$
S=\frac{1}{16 \pi G_{5}} \int d^{5} x \sqrt{-g}\left[R-2 \Lambda-F_{M N}^{a} F^{a M N}+\frac{S_{a b c}}{6 \sqrt{-g}} \varepsilon^{P K L M N} A_{P}^{a} F_{K L}^{b} F_{M N}^{c}\right],
$$

where $\Lambda=-6$ is the cosmological constant. As usual, the $U(1)$ field strengths are defined by $F_{M N}^{a}=\partial_{M} A_{N}^{a}-\partial_{N} A_{M}^{a}$, where $M, N, \ldots=0, \ldots, 4$ and $a=1, \ldots, n$. The Chern-Simons term $A \wedge F \wedge F$ encodes the information of the triangle anomalies in the field theory [ए]]. In fact, the Chern-Simons coefficients $S_{a b c}$ are related to the anomaly coefficients $C_{a b c}$ by

$$
C_{a b c}=S_{a b c} /\left(4 \pi G_{5}\right)
$$

The corresponding equations of motion are given by the combined system of Einstein-Maxwell and Maxwell equations,

$$
G_{M N}-6 g_{M N}=T_{M N}, \quad \nabla_{M} F^{a M P}=-\frac{S_{a b c}}{8 \sqrt{-g}} \varepsilon^{P M N K L} F_{M N}^{b} F_{K L}^{c}
$$

where the energy-momentum tensor $T_{M N}$ is

$$
T_{M N}=-2\left(F_{M R}^{a} F_{N}^{a R}+\frac{1}{4} g_{M N} F_{S R}^{a} F^{a S R}\right) .
$$

\subsection{AdS black hole with multiple U(1) charges}

A gravity dual to an isotropic fluid $(\varepsilon=3 P)$ with multiple chemical potentials $\mu^{a}(a=1, \ldots, n)$ at finite temperature $T$ is given by an AdS black hole solution with mass $m$ and multiple $U(1)$ charges $q^{a}$. In Eddington-Finkelstein coordinates, the metric and $U(1)$ gauge fields are

$$
d s^{2}=-f(r) d t^{2}+2 d r d t+r^{2} d \vec{x}^{2}, \quad A^{a}=-A_{0}^{a}(r) d t
$$

where

$$
f(r)=r^{2}-\frac{m}{r^{2}}+\sum_{a} \frac{\left(q^{a}\right)^{2}}{r^{4}}, \quad A_{0}^{a}(r)=\mu_{\infty}^{a}+\frac{\sqrt{3} q^{a}}{2 r^{2}} .
$$

In case of a single charge $(n=1)$, the background reduces to an ordinary Reissner-Nordstrøm black hole solution in $A d S_{5}$ [[25].

The temperature $T$ and chemical potentials $\mu^{a}$ of the fluid are defined by

$$
T=\frac{f^{\prime}\left(r_{+}\right)}{4 \pi}=\frac{2 r_{+}^{6}-\sum_{a}\left(q_{a}\right)^{2}}{2 \pi r_{+}^{5}}, \quad \mu^{a}=A_{0}^{a}\left(r_{\infty}\right)-A_{0}^{a}\left(r_{+}\right),
$$

where $r_{+}$is the outer horizon defined by the maximal solution of $f(r)=0$, and the boundary is located at $r_{\infty}$. The temperature of the fluid is the Hawking temperature of the black hole. 


\section{Holographic vortical and magnetic conductivities}

We will now compute the chiral vortical and magnetic conductivities $\xi_{\omega}^{a}$ and $\xi_{B}^{a b}$ from firstorder corrections to the AdS geometry (B.4) using the fluid-gravity correspondence [ [26].

\subsection{First-order corrected background and chiral conductivities}

In order to become a dual to a multiply-charged fluid, the AdS geometry (B.4) must be boosted along the four-velocity of the fluid $u_{\mu}(\mu=0, \ldots, 3)$. The boosted version of (B.4) is

$$
d s^{2}=\left(r^{2} P_{\mu v}-f(r) u_{\mu} u_{v}\right) d x^{\mu} d x^{v}-2 u_{\mu} d x^{\mu} d r, \quad A^{a}=\left(A_{0}^{a}(r) u_{\mu}+\mathscr{A}_{\mu}^{a}\right) d x^{\mu},
$$

where $P^{\mu v}=g^{\mu v}+u^{\mu} u^{v}$, and $f(r)$ and $A_{0}^{a}(r)$ as in (B.5). Following [ㅁ], [3], we have formally introduced constant background gauge fields $\mathscr{A}_{\mu}^{a}$ to model external electromagnetic fields, such as the magnetic fields $B^{a \mu}$ needed for the chiral magnetic effect.

The transport coefficients $\xi_{\omega}^{a}$ and $\xi_{B}^{a b}$ can now be computed using standard fluid-gravity techniques [[26]. We closely follow [ㄱ, [27, B], in which these transport coefficients were determined for an isotropic fluid with one and three charges $(n=1,3)$. We work in the static frame $u_{\mu}=(-1,0,0,0)$ and consider vanishing background fields $\mathscr{A}_{\mu}^{a}\left(\right.$ at $\left.x^{\mu}=0\right)$. The transport coefficients $\xi_{\omega}^{a}$ and $\xi_{B}^{a b}$ measure the response of the system to rotation and the perturbation by an external magnetic field. We therefore slowly vary $u_{\mu}$ and $\mathscr{A}_{\mu}^{a}$ up to first order as $(i=1,2,3)$

$$
u_{\mu}=\left(-1, x^{v} \partial_{v} u_{i}\right), \quad \mathscr{A}_{\mu}^{a}=\left(0, x^{v} \partial_{v} \mathscr{A}_{i}^{a}\right) .
$$

Due to the dependence on $x^{\mu}$, the background (4. 4 ) is no longer an exact solution of the equations of motion. Instead with varying parameters the solution (4-1) receives higher-order corrections, which are in this case of first order in the derivatives.

An ansatz for the first-order corrected metric and gauge fields is given by

$$
\begin{aligned}
d s^{2}= & \left(-f(r)+\tilde{g}_{t t}\right) d t^{2}+2\left(1+\tilde{g}_{t r}\right) d t d r+r^{2}\left(d x^{2}+d y^{2}+d z^{2}\right) \\
& +\tilde{g}_{i j} d x^{i} d x^{j}-2 x^{v} \partial_{v} u_{i} d r d x^{i}+2\left(\left(f(r)-r^{2}\right) x^{v} \partial_{v} u_{i}+\tilde{g}_{t i}\right) d t d x^{i}, \\
A^{a}= & \left(-A_{0}^{a}(r)+\tilde{A}_{t}^{a}\right) d t+\left(A_{0}^{a}(r) x^{v} \partial_{v} u_{i}+x^{v} \partial_{v} \mathscr{A}_{i}^{a}+\tilde{A}_{i}^{a}\right) d x^{i},
\end{aligned}
$$

where we denote the first-order corrections by $\tilde{g}_{M N}=\tilde{g}_{M N}(r)$ and $\tilde{A}_{M}^{a}=\tilde{A}_{M}^{a}(r)$ and choose the gauge $\tilde{g}_{r r}=0, \tilde{g}_{r \mu} \sim u_{\mu}, \tilde{A}_{r}^{a}=0, \sum_{i=1}^{3} \tilde{g}_{i i}=0$.

The first-order corrections can be obtained by substituting the ansatz (4.31) into the equations of motion (B.2). The computation is straight-forward but lengthy and can be found in appendix C

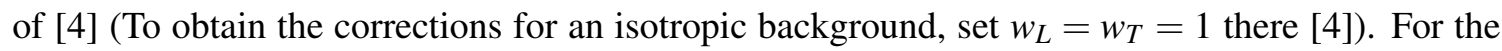
magnetic and vortical effects, we only need the gauge field corrections $\tilde{A}_{\mu}^{a}(r)$ given by

$$
\tilde{A}_{t}^{a}=0, \quad \tilde{A}_{i}^{a}(r)=\int_{\infty}^{r} d r^{\prime} \frac{1}{r^{\prime} f\left(r^{\prime}\right)}\left[Q_{i}^{a}\left(r^{\prime}\right)-Q_{i}^{a}\left(r_{+}\right)-C_{i} r_{+} A_{0}^{a \prime}\left(r_{+}\right)+r^{\prime} \tilde{g}_{t i}\left(r^{\prime}\right) A_{0}^{a \prime}\left(r^{\prime}\right)\right],
$$

where $Q_{i}^{a}$ and $C_{i}$ are defined by

$$
Q_{i}^{a} \equiv \frac{1}{2} S^{a b c} A_{0}^{b} A_{0}^{c} \varepsilon^{i j k}\left(\partial_{j} u_{k}\right)+S^{a b c} A_{0}^{b} \varepsilon^{i j k}\left(\partial_{j} \mathscr{A}_{k}^{c}\right)
$$




$$
\begin{gathered}
C_{i}=4 c\left(r_{+}\right)\left(\frac{1}{3} S^{a b c} A_{0}^{a}\left(r_{+}\right) A_{0}^{b}\left(r_{+}\right) A_{0}^{c}\left(r_{+}\right) \varepsilon^{i j k}\left(\partial_{j} u_{k}\right)+\frac{1}{2} S^{a b c} A_{0}^{a}\left(r_{+}\right) A_{0}^{b}\left(r_{+}\right) \varepsilon^{i j k}\left(\partial_{j} \mathscr{A}_{k}^{c}\right)\right), \\
c\left(r_{+}\right)=\left[r_{+}\left(f^{\prime}\left(r_{+}\right)-4 \sum_{a} A_{0}^{a}\left(r_{+}\right) A_{0}^{a \prime}\left(r_{+}\right)\right)\right]^{-1} .
\end{gathered}
$$

The term involving $\tilde{g}_{t i}\left(r^{\prime}\right)$ will not be needed in the following.

On the boundary of the asymptotic AdS space (4.3)), the metric and gauge fields couple to the fluid stress-energy tensor and $U(1)$ currents, respectively. Holographic renormalization [28] provides relations between these currents and the near-boundary behavior of their dual bulk fields. The $U(1)$ currents $j^{a \mu}$ are related to the bulk gauge fields $A^{a \mu}$ by [28, [2R]

$$
j^{a \mu}=\frac{1}{2 \pi G_{5}}\left[\eta^{\mu v} A_{v}^{a(2)}-\hat{j}^{a \mu}\right], \quad \hat{j}^{a \mu} \propto S^{a b c} \varepsilon^{\mu v \rho \sigma} A_{v}^{b(0)} \partial_{\rho} A_{\sigma}^{c(0)},
$$

where $A_{\mu}^{a(n)}$ are the $n$ th-order coefficients in a $\frac{1}{r}$-expansion of the bulk gauge fields $A_{\mu}^{a}\left(r, x^{\mu}\right)$. Setting $A_{v}^{a(0)}=\mu_{\infty}^{a} u_{v}=0$ allows us to ignore contributions from $\hat{j}^{a \mu}$.

Expanding the solution in $\frac{1}{r}$ and substituting only the corrections $\tilde{A}_{\mu}^{a}$, we get the currents

$$
\tilde{j}^{a \mu}=\lim _{r \rightarrow \infty} \frac{r^{2}}{2 \pi G_{5}} \eta^{\mu v} \tilde{A}_{v}^{a}(r)=\frac{1}{4 \pi G_{5}} \eta^{\mu v}\left(Q_{v}^{a}\left(r_{+}\right)+r_{+} A_{0}^{a \prime}\left(r_{+}\right) C_{v}\right) .
$$

Substituting (B.5) into the right-hand side of (4.6) and comparing the result with ${ }^{3}$

$$
\tilde{j}^{a \mu}=\xi_{\omega}^{a} \omega^{\mu}+\xi_{B}^{a b} B^{b \mu}=\xi_{\omega}^{a} \frac{1}{2} \varepsilon^{v \rho \sigma \mu} u_{v} \partial_{\rho} u_{\sigma}+\xi_{B}^{a b} \varepsilon^{v \rho \sigma \mu} u_{v} \partial_{\rho} \mathscr{A}_{\sigma}^{b},
$$

we finally obtain the coefficients

$$
\begin{aligned}
& \xi_{\omega}^{a}=\frac{1}{4 \pi G_{5}}\left(S^{a b c} \mu^{b} \mu^{c}-\frac{2}{3} \frac{\rho^{a}}{\varepsilon+P} S^{b c d} \mu^{b} \mu^{c} \mu^{d}\right), \\
& \xi_{B}^{a b}=\frac{1}{4 \pi G_{5}}\left(S^{a b c} \mu^{c}-\frac{1}{2} \frac{\rho^{a}}{\varepsilon+P} S^{b c d} \mu^{c} \mu^{d}\right),
\end{aligned}
$$

with $\mu^{a} \equiv-A_{0}^{a}\left(r_{+}\right)$(since $A_{0}^{a}\left(r_{\infty}\right)=\mu_{\infty}^{a}=0$ ), in agreement with ([2.4) and (ㄷ.5). Restricting to two charges $(n=2)$ and performing similar manipulations as in section [2.2, we recover the chiral conductivities $\kappa_{\omega}$ and $\kappa_{B}$ given by ([2.12).

\subsection{Subtleties in holographic descriptions of the CME}

The conservation of the electromagnetic current requires the introduction of the Bardeen counterterm into the action. In AdS/QCD models of the CME, this typically leads to a vanishing

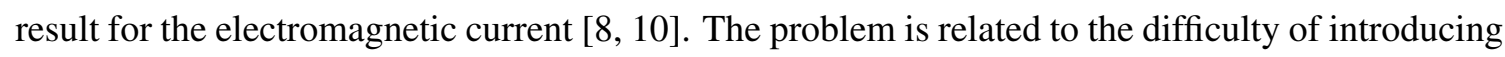
a chemical potential conjugated to a non-conserved chiral charge [ [8, Q 0$]$. It is possible to modify the action to obtain a conserved chiral charge [ [8]. This charge is however only gauge invariant when integrated over all space in homogeneous configurations.

\footnotetext{
${ }^{3}$ Use also $4 r_{+} A_{0}^{a \prime}\left(r_{+}\right) c\left(r_{+}\right)=\sqrt{3} q^{a} / m=\rho^{a} /(\varepsilon+P)$ for the prefactor of the second term in (4.6) [四] (We thereby correct a factor 4 in [䧃]. Note that $\left.\rho^{a}=j^{a 0}=\sqrt{3} q^{a} /\left(4 \pi G_{5}\right)\right)$.
} 
In AdS black hole models of the CME, one usually introduces a chiral chemical potential dual to a gauge invariant current, despite it being anomalous [Q [0, []]. Consider the physical case with two charges $(n=2)$ as in section 2.2 and define axial and vector gauge fields by $A_{\mu}^{A}=A_{\mu}^{1}$ and $A_{\mu}^{V}=A_{\mu}^{2}$. Then $\hat{j}^{\mu}=\hat{j}_{2}^{\mu}$ gives rise to an additional contribution of the type

$$
\hat{j}^{\mu} \supset \varepsilon^{\mu \nu \rho \sigma} A_{v}^{A(0)}(x) F_{\rho \sigma}^{V(0)}(x),
$$

which is however forbidden by electromagnetic gauge invariance [8]. This forces us to set $A_{v}^{A(0)}(x)=$ $\mu_{5}^{\infty} u_{v}=0($ at $x=0)$. However, the chiral chemical potential $\mu_{5}=A_{0}^{A}\left(r_{\infty}\right)-A_{0}^{A}\left(r_{+}\right)=\mu_{5}^{\infty}-A_{0}^{A}\left(r_{+}\right)$ should be non-vanishing. Since $\mu_{5}^{\infty}=0, A_{0}^{A}\left(r_{+}\right)$must be non-vanishing, $A_{0}^{A}\left(r_{+}\right) \neq 0$, corresponding to a non-vanishing gauge field at the horizon. In a static charged AdS black hole solution, one should set $A_{0}^{A}\left(r_{+}\right)=0$ in order to get a well-defined gauge field at the horizon [[25]. However, this is not necessarily required in the fluid-gravity duality, see [22], in particular footnote 5 therein, and $A_{0}^{A}\left(r_{+}\right) \neq 0$ does not lead to an inconsistency.

\section{CME in anisotropic fluids}

The above analysis can be repeated for anisotropic fluids with (rest-frame) stress-energy tensor $T^{\mu v}=\operatorname{diag}\left(\varepsilon, P_{T}, P_{T}, P_{L}\right)$ and different pressures $P_{T} \neq P_{L}$, see [䧃] for details. It is expected that in this case the chiral magnetic conductivity $\kappa_{B}$ may depend on the anisotropy parameter $\varepsilon_{p}=\frac{P_{T}-P_{L}}{P_{T}+P_{L}}$. In fact, for small anisotropies $\varepsilon_{p}$, we found (average pressure $\bar{P}=\left(2 P_{T}+P_{L}\right) / 3$ ) [四]

$$
\kappa_{B} \approx C \mu_{5}\left(1-\frac{\mu \rho}{\varepsilon+\bar{P}}\left[1-\frac{\varepsilon_{p}}{6}\right]\right) .
$$

Despite the crudeness of the model, one can assume that such an anisotropic fluid describes to some extent the anisotropic quark-gluon plasma, with our $\varepsilon_{p}$ imitating the real $\varepsilon_{p} \approx 2 v_{2}$ for pions. Since the net chemical potential $\mu$ is quite small in current heavy-ion experiments, the dependence on $\varepsilon_{P}$ (and hence $v_{2}$ ) in (5.J) appears to be very mild. Even though the anisotropy dependence of $\kappa_{B}$ is very weak, (ل. (ل) tells us how the CME, if present in the experimental data, can be separated from the $v_{2}$-dependent background (for one of the attempts of such a separation see [[]]).

\section{References}

[1] D. Kharzeev, Phys. Lett. B 633, 260 (2006) [arXiv:0406125] • D. Kharzeev and A. Zhitnitsky, Nucl. Phys. A 797, 67 (2007) [arXiv:0706.1026] • D. E. Kharzeev, L. D. McLerran and H. J. Warringa, Nucl. Phys. A 803, 227 (2008) [arXiv:0711.0950] • K. Fukushima, D. E. Kharzeev and H. J. Warringa, Phys. Rev. D 78, 074033 (2008) [arXiv:0808.3382].

[2] A. Bzdak, V. Koch and J. Liao, arXiv:1207.7327 [nucl-th].

[3] T. Kalaydzhyan and I. Kirsch, Phys. Rev. Lett. 106 (2011) 211601 [arXiv:1102.4334].

[4] I. Gahramanov, T. Kalaydzhyan and I. Kirsch, Phys. Rev. D 85, 126013 (2012) [arXiv:1203.4259 [hep-th]].

[5] G. Lifschytz and M. Lippert, Phys. Rev. D 80, 066005 (2009) [arXiv:0904.4772].

[6] H. U. Yee, JHEP 0911, 085 (2009) [arXiv:0908.4189]. 
[7] A. Gorsky, P. N. Kopnin and A. V. Zayakin, Phys. Rev. D 83, 014023 (2011) [arXiv:1003.2293].

[8] V. A. Rubakov, [arXiv:1005.1888].

[9] A. Gynther, K. Landsteiner, F. Pena-Benitez and A. Rebhan, JHEP 1102, 110 (2011) [arXiv:1005.2587].

[10] A. Rebhan, A. Schmitt and S. A. Stricker, JHEP 1001, 026 (2010) [arXiv:0909.4782].

[11] L. Brits and J. Charbonneau, Phys. Rev. D 83, 126013 (2011) [arXiv:1009.4230].

[12] I. Amado, K. Landsteiner and F. Pena-Benitez, JHEP 1105, 081 (2011) [arXiv:1102.4577].

[13] C. Hoyos, T. Nishioka and A. O'Bannon, JHEP 1110, 084 (2011) [arXiv:1106.4030 [hep-th]].

[14] J. Bhattacharya, S. Bhattacharyya, S. Minwalla and A. Yarom, [arXiv:1105.3733].

[15] K. Landsteiner and L. Melgar, JHEP 1210, 131 (2012) [arXiv:1206.4440 [hep-th]].

[16] K. Landsteiner, E. Megias and F. Pena-Benitez, arXiv:1207.5808 [hep-th].

[17] D. T. Son and P. Surowka, Phys. Rev. Lett. 103, 191601 (2009) [arXiv:0906.5044].

[18] A. V. Sadofyev, V. I. Shevchenko and V. I. Zakharov, Phys. Rev. D 83 (2011) 105025 [arXiv:1012.1958].

[19] A. V. Sadofyev and M. V. Isachenkov, Phys. Lett. B 697, 404 (2011) [arXiv:1010.1550].

[20] S. Pu, J. h. Gao and Q. Wang, Phys. Rev. D 83, 094017 (2011) [arXiv:1008.2418] • J. -H. Gao, Z. -T. Liang, S. Pu, Q. Wang and X. -N. Wang, [arXiv:1203.0725].

[21] Y. Neiman and Y. Oz, JHEP 1103, 023 (2011) [arXiv:1011.5107].

[22] J. Erdmenger, M. Haack, M. Kaminski and A. Yarom, JHEP 0901, 055 (2009) [arXiv:0809.2488].

[23] K. Landsteiner, E. Megias and F. Pena-Benitez, Phys. Rev. Lett. 107, 021601 (2011) [arXiv:1103.5006 [hep-ph]].

[24] D. E. Kharzeev and D. T. Son, Phys. Rev. Lett. 106, 062301 (2011) [arXiv:1010.0038].

[25] A. Chamblin, R. Emparan, C. V. Johnson and R. C. Myers, Phys. Rev. D 60, 104026 (1999) [arXiv:hep-th/9904197].

[26] S. Bhattacharyya, V. E. Hubeny, S. Minwalla and M. Rangamani, JHEP 0802, 045 (2008) [arXiv:0712.2456].

[27] M. Torabian and H. U. Yee, JHEP 0908, 020 (2009) [arXiv:0903.4894].

[28] M. Bianchi, D. Z. Freedman and K. Skenderis, Nucl. Phys. B 631, 159 (2002) [arXiv:hep-th/0112119].

[29] B. Sahoo and H. U. Yee, JHEP 1011, 095 (2010) [arXiv:1004.3541]. 